\title{
Impact of COVID-19 on gastric cancer treatment in Japanese high-volume centers: a JCOG stomach cancer study group survey
}

\author{
Masanori Tokunaga ${ }^{1} \cdot$ Takaki Yoshikawa $^{2} \cdot$ Narikazu Boku $^{3} \cdot$ Yasunori Nishida $^{4} \cdot$ Toshiyuki Tanahashi $^{5}$. \\ Takanobu Yamada $^{6} \cdot$ Shusuke Haruta $^{7} \cdot$ Tsuyoshi Etoh $^{8} \cdot$ Noriyuki Hirahara $^{9} \cdot$ Yasuyuki Kawachi $^{10} \cdot$ Kunihiro Tsuji $^{11}$. \\ Takahiro Kinoshita $^{12} \cdot$ Takashi Kanazawa $^{13} \cdot$ Noriaki Tokumoto $^{14}$. Junya Fujita ${ }^{15} \cdot$ Masanori Terashima $^{16}$
}

Received: 28 January 2021 / Accepted: 1 May 2021 / Published online: 20 July 2021

(c) Springer Nature Singapore Pte Ltd. 2021

\begin{abstract}
Purposes The spread of coronavirus disease 2019 (COVID-19) has affected socioeconomic and healthcare systems in many countries. Accordingly, many individuals may have canceled their annual health-check programs, including esophagogastroduodenoscopy, which would have resulted in lower numbers of newly diagnosed patients with gastric cancer in comparison to other times.

Methods Questionnaires were distributed to 62 hospitals every week from May 2020 to August 2020 (total 744) through mailing lists of the Stomach Cancer Study Group of the Japan Clinical Oncology Group. The number of patients with gastric cancer and hospital systems during the COVID-19 pandemic were surveyed.

Results In total, 74\% (551 out of 744) of the questionnaires were answered and analyzed. In early May, approximately 50\% of hospitals had to restrict surgical slots due to the COVID-19 pandemic. However, they gradually loosened the restrictions thereafter. The number of gastrectomies was $<80 \%$ that of the same period in the previous year, and hospitals in Tokyo were seriously affected by a $50 \%$ decrease in the number of gastrectomies.

Conclusions The number of gastrectomies was lower than that in the previous year. Further multi-center follow-up studies are required to evaluate the long-term effects of COVID-19 on the clinical outcomes of patients with gastric cancer.
\end{abstract}

Keywords Gastrectomy $\cdot$ Coronavirus $\cdot$ COVID-19 $\cdot$ Stomach neoplasms $\cdot$ Socioeconomic factors

Masanori Tokunaga

tokunaga.srg1@tmd.ac.jp

1 Department of Gastrointestinal Surgery, Tokyo Medical and Dental University, 1-5-45 Yushima, Bunkyo-ku, Tokyo, Japan

2 Division of Gastric Surgery, National Cancer Center Hospital, Tokyo, Japan

3 Division of Gastrointestinal Medical Oncology, National Cancer Center Hospital, Tokyo, Japan

4 Department of Surgery, Keiyukai Sapporo Hospital, Sapporo, Japan

5 Department of Surgery, Gifu Municipal Hospital, Gifu, Japan

6 Department of Gastrointestinal Surgery, Kanagawa Cancer Center, Kanagawa, Japan

7 Department of Gastroenterological Surgery, Toranomon Hospital, Tokyo, Japan

8 Department of Gastroenterological and Pediatric Surgery, Oita University Faculty of Medicine, Oita, Japan
9 Department of Digestive and General Surgery, Shimane University Faculty of Medicine, Shimane, Japan

10 Department of Surgery, Nagaoka Chuo General Hospital, Nagaoka, Japan

11 Departments of Gastroenterology, Ishikawa Prefectural Central Hospital, Ishikawa, Japan

12 Department of Gastric Surgery, National Cancer Center Hospital East, Kashiwa, Japan

13 Department of Surgery, Fukuyama City Hospital, Hiroshima, Japan

14 Department of Surgery, Hiroshima City Asa Citizens Hospital, Hiroshima, Japan

15 Department of Surgery, Sakai City Medical Center, Osaka, Japan

16 Division of Gastric Surgery, Shizuoka Cancer Center, Shizuoka, Japan 


\section{Introduction}

The incidence of gastric cancer is high in East Asia and more than half of the diagnosed cases are diagnosed in the early stage in Japan and Korea [1,2]. Regular screening programs provided by local governments and easy access to esophagogastroduodenoscopy (EGD) procedures are major reasons for the earlier detection of gastric cancer in Japan and Korea in comparison to Western countries [3].

At the end of 2019, the first case of coronavirus disease 2019 (COVID-19) was reported in Wuhan, China. Since then, the spread of COVID-19 has affected the socioeconomic and healthcare systems of many countries, including Japan (Supplementary Fig. 1) [4-9]. Elderly people are reportedly more susceptible to COVID-19 with higher mortality rates. Therefore, "stay-at-home orders" urging people to stay home as much as possible has heavily affected the lifestyle of this population [10]. Although less susceptible, younger people were also asked to stay home to avoid infection and to stop the spread of COVID-19. Since it is considered that visiting a hospital increases the risk of COVID-19 transmission, many people have refrained from going to hospitals unless they are seriously ill. Accordingly, many people might have canceled their annual health-check programs, including EGD, which would result in smaller numbers of new gastric cancer diagnoses in comparison to usual years [11].

A possible consequence might be that early gastric cancers that could have been curatively resected by endoscopic resection would progress to an advanced stage that requires extensive surgery. Moreover, resectable gastric cancer may metastasize and progress to a stage for which curative gastrectomy would no longer be indicated. If the number of newly diagnosed cases of gastric cancer at regular check-ups is actually decreasing, we need to take actions to encourage people to receive their check-ups, particularly if they have upper gastrointestinal tract symptoms. However, the extent of the decrease in the number of patients who could be newly diagnosed with gastric cancer since the beginning of the COVID-19 pandemic is unclear and the impact of COVID-19 on clinical oncology remains to be elucidated. To shed light on these issues, we conducted a questionnaire survey to assess the impact of the COVID-19 pandemic on gastric cancer treatment.

\section{Patients and methods}

A questionnaire was distributed through a mailing list of the Stomach Cancer Study Group of the Japan Clinical Oncology Group (JCOG), in which researchers from 62 participating hospitals were included. The first questionnaire was sent on April 30, 2020, and follow-up questionnaires were sent every week until the second week of June, and every 2 weeks thereafter. The final questionnaire was sent on August 24. Coordinators of each institute answered the questionnaires. If a single coordinator inadvertently answered one questionnaire multiple times, the earliest one was used for the analysis.

The content of the questionnaires is shown in Fig. 1. With the exception of the first two questionnaires, which did not include question regarding the number of gastrectomies performed per a week, all items were included in each questionnaire. To comprehensively assess the effects of COVID-19, the questionnaire included the effects of COVID-19 on the number of patients diagnosed with gastric cancer $(\mathrm{Q} 1, \mathrm{Q} 2)$, the hospital system (Q3-Q5), surgery (Q6-Q8), chemotherapy (Q9, Q10), endoscopy (Q11, Q12), and clinical trials (Q13).

Microsoft Form was used to distribute questionnaires to researchers and to collect their answers.

As this survey did not have access to individual patient data, and all questionnaires were conducted on a voluntary basis, the requirement for Institutional Review Board approval was waived.

\section{Estimation of the effects of COVID-19 on the number of gastrectomies}

The expected number of gastrectomies per week prior to the COVID-19 pandemic was calculated by dividing the number of gastrectomies in 2019 by 52 (weeks). The ratio of the actual number in each week to the expected number was calculated thereafter.

\section{Categorization of institutions}

Institutions were categorized according to the location and type. The location was categorized into the Kanto area, which includes Tokyo (the most populated area in Japan), the Kansai area, which includes Osaka (the second most populated area), and other areas. Institutions were classified as university hospitals, cancer specialized centers, and general hospitals.

\section{Results}

In Japan, a state of emergency was initially declared on April 7, 2020 in seven prefectures where the number of COVID19-infected patients was high. On April 16, 2020, the state of emergency was expanded to the whole country and lasted for approximately 1 month until May 14, 2020, when the state of emergency was lifted in 39 prefectures. It was completely 
Q1. How many gastrectomies for gastric cancer were performed last week?

Q2. How much has the number of new outpatients with gastric cancer changed compared to that before the COVID-19 outbreak?

$\begin{array}{llll}\text { 1. slightly increased } & \text { 2. not changed } & \text { 3. slightly decreased } & 4 \text {. drastically decreased }\end{array}$

Q3. Has your institution received COVID-19 patients in the last two weeks?

$\begin{array}{ll}\text { 1. YES } & \text { 2. NO }\end{array}$

Q4. Has a COVID-19 outbreak been seen in your institution in the last two weeks?

$\begin{array}{ll}\text { 1. YES } & \text { 2. NO }\end{array}$

Q5. Are surgeons, medical oncologists, or endoscopists who take care of gastric cancer patients forced to treat patients with COVID-19?

1. YES 2. NO

Q6. Are surgical slots for malignant disease restricted due to COVID-19? If so, how much?

$\begin{array}{lllll}\text { 1. No restriction } & \text { 2. } 1-33 \% \text { restriction } & \text { 3. } 34-66 \% \text { restriction } & 4.67-99 \% \text { restriction } & 5.100 \% \text { restriction }\end{array}$

Q7. Are surgical slots for benign disease restricted due to COVID-19? If so, how much?

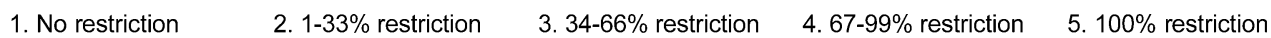

Q8. Is emergency surgery restricted due to COVID-19?

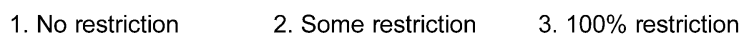

Q9. Is inpatient chemotherapy restricted due to COVID-19? If so, how much?

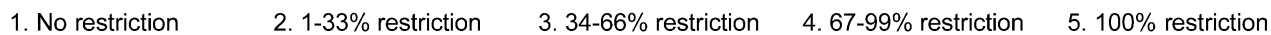

Q10. Is outpatient chemotherapy restricted due to COVID-19? If so, how much?

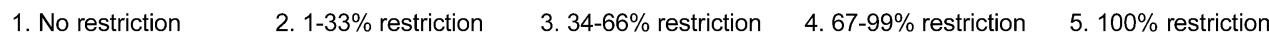

Q11. Is endoscopic examination restricted due to COVID-19? If so, how much?

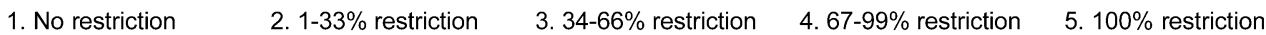

Q12. Is endoscopic treatment (endoscopic submucosal dissection) restricted due to COVID-19? If so, how much?

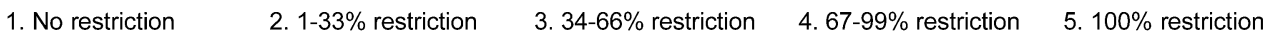

Q13. Is registration to clinical trials restricted due to COVID-19? If so, how much?

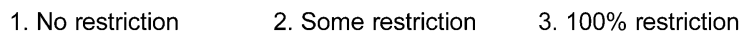

Fig. 1 The contents of the questionnaire survey

lifted on May 25, 2020 (Supplementary Fig. 2). Supplementary Fig. 1 shows the number of COVID-19 patients in Japan since January 2020. The number gradually dropped during the state of emergency. However, it increased again thereafter. During the state of emergency, people were urged to stay-at-home, and many facilities, including schools, restaurants, department stores other than grocery shops, and event halls, were also asked to close.

The JCOG Stomach Cancer Study Group includes 20 university hospitals, 15 cancer specialized centers, and 27 general hospitals. The number of hospitals in each area was as follows: Kanto ( $n=14$; including 6 hospitals in Tokyo), Kansai $(n=17)$, and other areas $(n=31)$ (Supplementary Fig. 3).

In total, the questionnaire was sent 12 times in 17 weeks to 62 institutions, and all institutions responded to the questionnaires at least once. Overall, among 744 questionnaires, $551(74 \%)$ replies were answered and analyzed (Supplementary Fig. 4). To obtain as many answers as possible to Q1, which was essential to estimate the actual decrease in the number of gastrectomies during the COVID-19 pandemic and which was considered one of the most important items in the questionnaire, a reminder was sent to the researchers in the second week of September, and they were asked to fill in the number of gastrectomies per each week during the study period. At the same time, they were also asked about the number of gastrectomy procedures in the previous year. In 2019, 6089 gastrectomies were performed in 49 institutions.

Figure 2 illustrates the effect of COVID-19 on the number of patients newly diagnosed with gastric cancer (Q2), the hospital system (Q3, Q5), and clinical trials (Q13). In the first week of this study, in approximately $50 \%$ of institutions, there was a decrease in the number of new patients, and the proportion gradually increased, reaching $80 \%$ during the last week of the study. Additionally, the proportion of institutions that experienced a drastic decrease in the number of new patients sharply increased in the early half of the study period, reaching approximately $50 \%$. Approximately half of the institutions hospitalized COVID-19 patients at the beginning of the study (the 1st week of May), gradually decreasing to $20 \%$ by the midway point (the $3 \mathrm{rd}$ and 4 th week of June). Finally, it increased again to $60 \%$ at the end of the study period (4th week of August). A similar trend could be seen in the answers to Q5.

Figure 3 shows the effect of COVID-19 on surgical slots. In the early study period (the 1 st to 4 th week of May), surgeries for malignant disease, including gastrectomy for gastric cancer, were restricted to approximately $40 \%$ of institutions, while those for benign disease, such as cholecystectomy and inguinal hernia repair, were restricted to approximately $60 \%$ of institutions. The degree of restriction was more severe for benign disease. Additionally, $20 \%$ of institutions had to restrict surgical slots by 

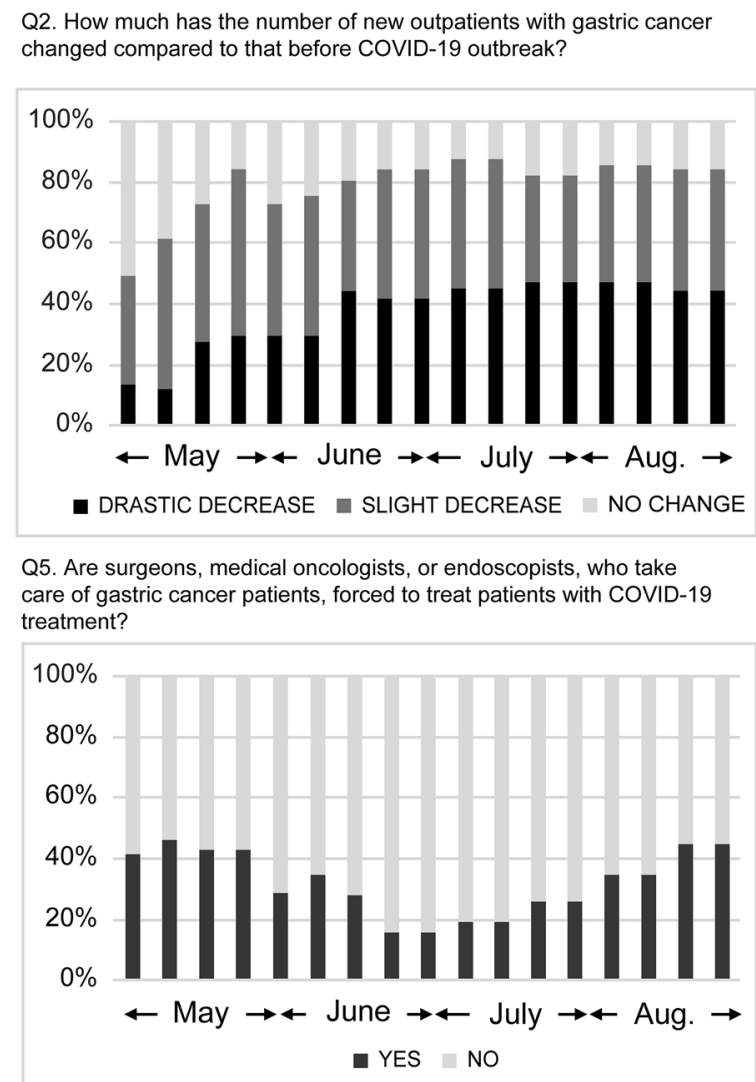

Q3. Has your institution received COVID-19 patients in the last two weeks?

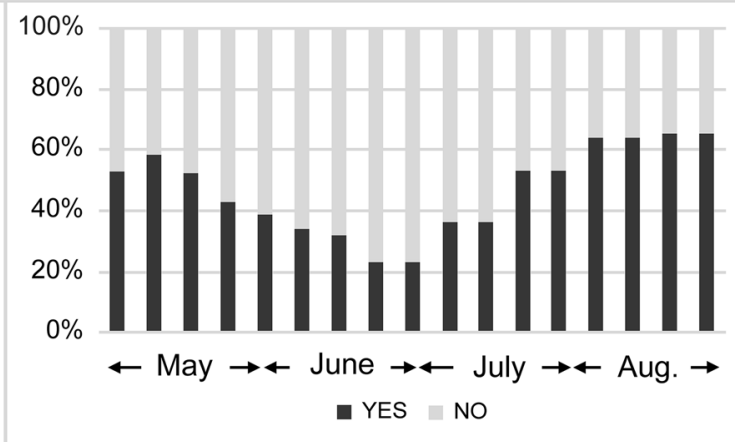

Q13. Is registration to clinical trials restricted due to COVID-19? If so, how much?

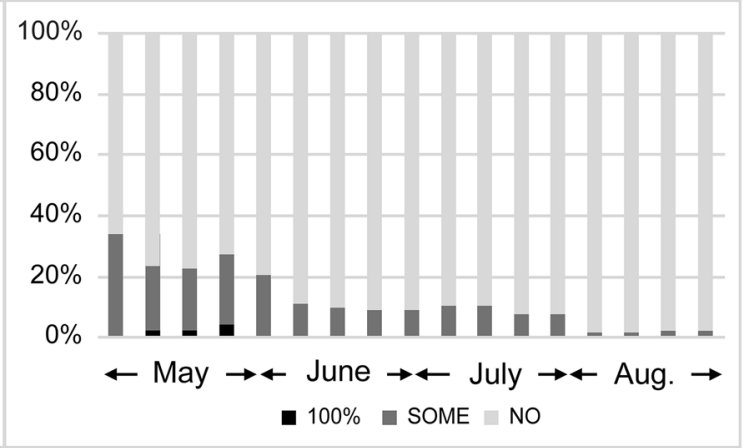

Fig. 2 The effect of COVID-19 on the number of patients (Q2), hospital system (Q3, Q5), and clinical trials (Q13)

more than $67 \%$ in comparison to normal times, while the degree of restriction for malignant disease was $<33 \%$ in comparison to normal times in most hospitals. The restrictions gradually weakened, and fewer than $20 \%$ of hospitals had mild restrictions (1-33\% of restriction) by the end of the study period. Emergency surgeries were less affected by COVID-19 than were elective surgeries.

Figure 4 depicts the effect of COVID-19 on chemotherapy and endoscopy, in which a similar trend to that in surgery was seen. Chemotherapy seemed to be less affected in comparison to surgery or endoscopy.

The number of gastrectomies during the study period was $<80 \%$ that of the previous year (Fig. 5). Hospitals located in areas other than Kanto and Kansai were less affected. Hospitals in Tokyo were seriously affected, and the number of gastrectomies was approximately $50 \%$ less than the number in the previous year. The type of institution also affected the number of gastrectomies: in which cancer centers were more strongly affected in comparison to university and general hospitals. During the study period, no one performed gastrectomy for patients with active and/or past COVID-19 infection.

\section{Discussion}

This questionnaire survey aimed to evaluate the effect of COVID-19 on daily practice for gastric cancer. It found that COVID-19 affected the number of gastrectomies, particularly in populated areas, with the most severe decrease in Tokyo, the capital of Japan.

Although the infection and mortality rates associated with COVID-19 in Japan have been low in comparison to Western countries, many hospitals have received COVID19 patients. Therefore, surgeons, medical oncologists, and endoscopists who usually take care of gastric cancer patients are involved in treating patients with COVID-19 [6]. In Japan, the government declared a state of emergency in early April 2020, and thereafter, the number of patients with COVID-19 was gradually decreased [12]. However, the number of patients began to increase again in the middle of July, and the proportion of hospitals in which surgeons, medical oncologists, or endoscopists were involved in the treatment of COVID-19 also started to increase. 


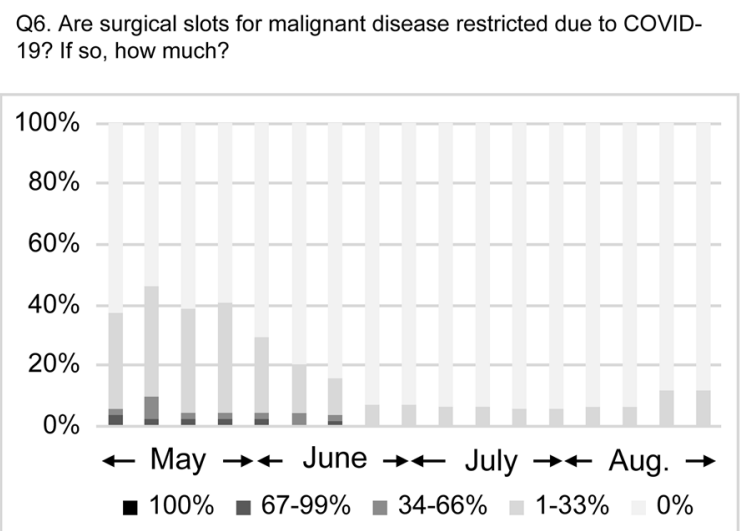

Q7. Are surgical slots for benign disease restricted due to COVID-19? If so, how much?

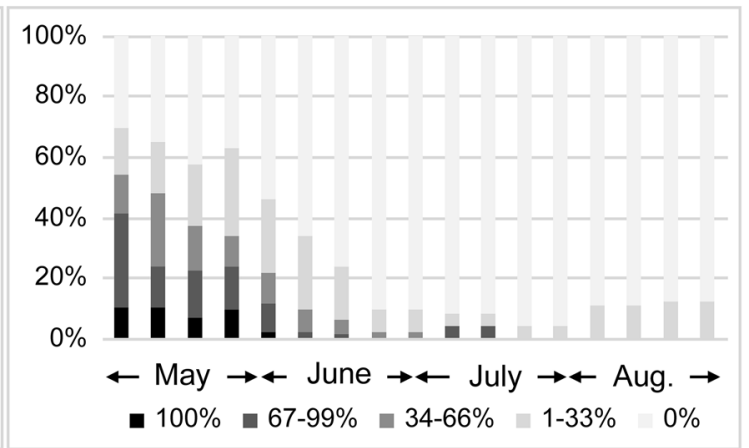

Q8. Is emergency surgery restricted due to COVID-19?

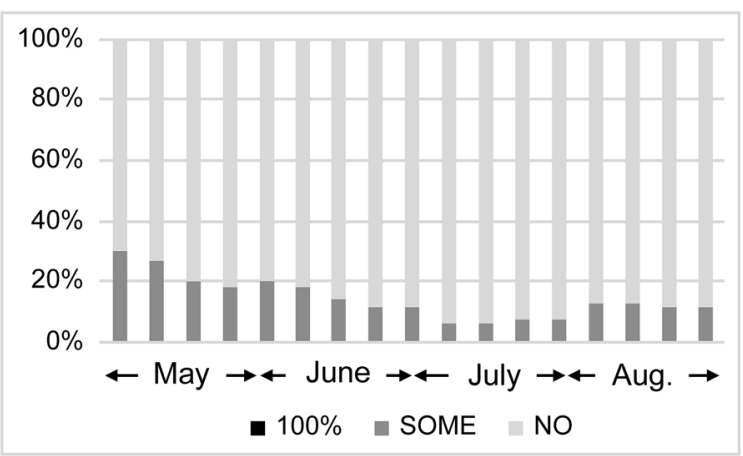

Fig. 3 The effect of COVID-19 on surgery for gastric cancer (Q6), benign disease (Q7), and emergency cases (Q8)

Surgery, chemotherapy, and endoscopy slots were restricted, to some extent, at the beginning of the pandemic, although these restrictions were gradually reduced. Initially, many hospitals seemingly prepared for the management of COVID-19 by restricting treatment for other patients, and thus had to limit surgical slots. On the other hand, hospitals did not need to restrict the number of slots in the latter period. However, even in the latter study period when the restriction of surgical slots was attenuated-in many hospitals, the number of gastric cancer patients was lower in comparison to the previous year (Q2 in Fig. 2). To estimate the decrease in gastrectomies, the ratio of the actual number of gastrectomies per week to that in the previous year was calculated. The number of gastrectomies was drastically decreased in the Kanto and Kansai areas, where the incidence of COVID-19 was higher in comparison to other regions. Notably, the number of gastrectomies in Tokyo, where COVID-19 patient numbers were the highest in Japan, decreased by $50 \%$ in comparison to the previous year. However, we have to note that this decrease might have been somewhat affected by the natural decrease in the number of patients with gastric cancer. Nevertheless, the drop by $50 \%$ in Tokyo cannot be explained without the effect of COVID19. The answer to this question will be obtained after the pandemic ends.

In Japan, local governments offer annual medical checkup programs for gastric cancer to residents of $\geq 50$ years of age. Additionally, if people have upper gastrointestinal tract symptoms, such as nausea or appetite loss, they can easily undergo EGD examination, which is partially reimbursed by national insurance. However, during the COVID19 pandemic, people in this age group, particularly those over 70 years of age, who were considered to be susceptible to COVID-19 infection, might have refrained from going to hospitals to receive medical check-ups, including EGD. This caused a decrease in the number of newly diagnosed patients. Furthermore, some local governments stopped or suspended offering EGD screening during the pandemic, which also caused a further decrease in the number of gastrectomies.

EGD is generally considered a high-risk procedure in terms of COVID-19 transmission, and people may avoid 


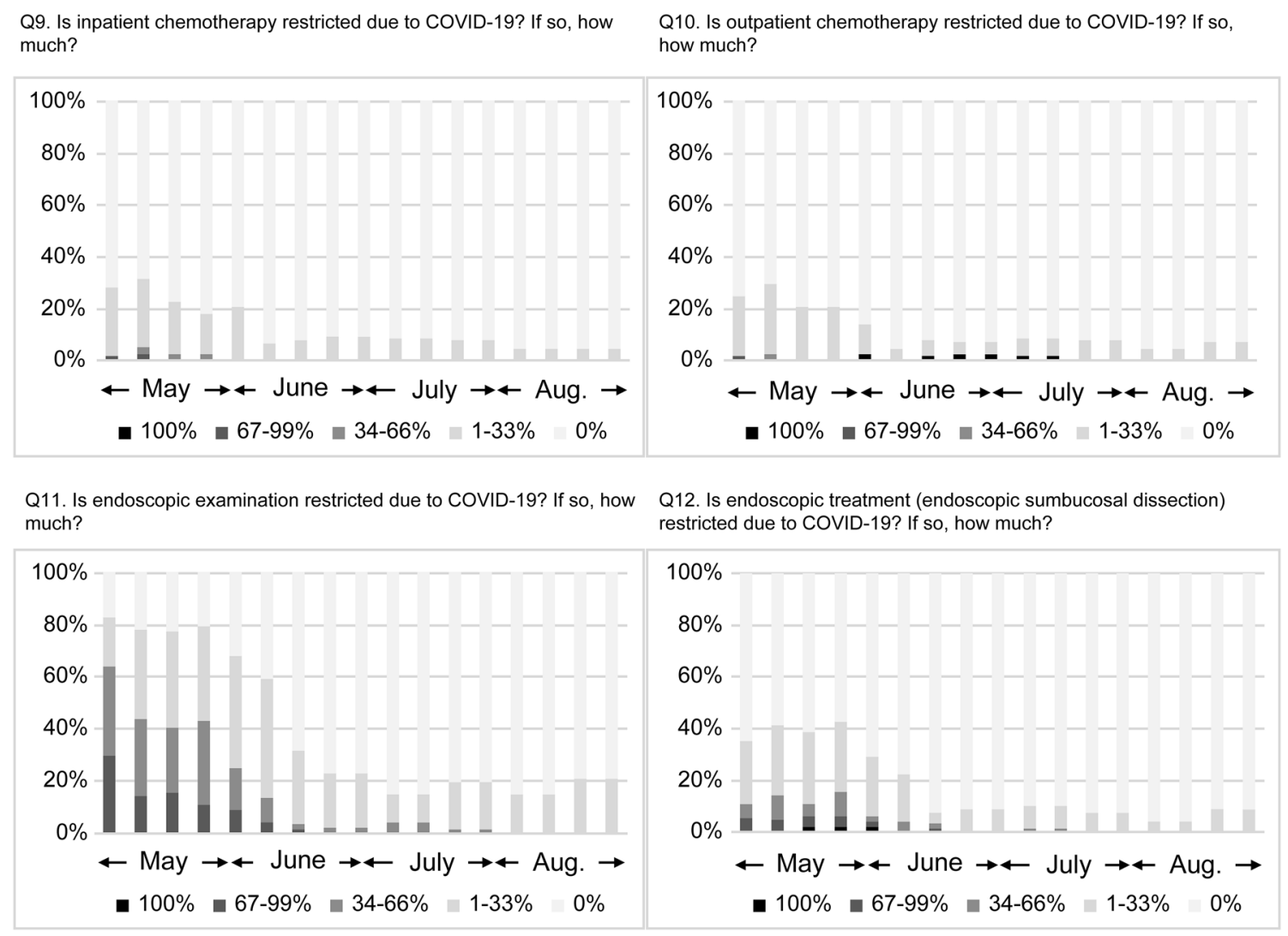

Fig. 4 The effect of COVID-19 on chemotherapy $(\mathrm{Q} 9,10)$ and endoscopy $(\mathrm{Q} 11,12)$

receiving EGD. Avoidance of EGD screening seemingly had a greater effect than surgical slot restriction, because the number of gastrectomies remained low, even after the attenuation of surgical slot restriction. To avoid the fatal consequences of curable gastric cancers becoming non-curable, we should encourage patients not to skip regular medical check-ups or hesitate to come to hospitals, even when the next pandemic occurs. However, we will have to wait for several years to finally evaluate the impact of COVID-19 in 2020, as the long-term survival outcomes are a vital parameter from the oncological aspect.

This questionnaire survey targeted hospitals affiliated with the JCOG gastric cancer group, and the locations and types of these hospitals were well balanced. Additionally, the number of gastrectomies performed at institutions included in this survey was approximately 6000 in 2019, which accounted for more than $10 \%$ of all cases in Japan. However, the results should be carefully interpreted as the hospitals included in this study are leading hospitals in gastric cancer care and treatment and thus may not accurately reflect the real-world situation during the COVID-19 pandemic in Japan. An analysis using a much larger national database is required in the future. However, to distribute up-to-date information to the public, the present questionnaire survey of selected hospitals seems worthwhile.

The present study was associated with some limitations, including the difficulty in applying the results to other countries. As the effects of COVID-19 differ among countries, each country needs to conduct their own original survey to assess their situation [13]. Another limitation is that this survey mainly focused on surgery for gastric cancer but not on endoscopic resection or chemotherapy in detail. Thus far, there have been a few reports about the number of patients receiving endoscopic resection or palliative chemotherapy during the COVID-19 pandemic. These issues therefore need to be evaluated and clarified in another study $[14,15]$.

The present questionnaire survey demonstrated the negative effect of COVID-19 on gastric cancer treatment in Japanese high-volume centers. The establishment of a medical system which can maintain the routine activity under precaution against COVID-19 and efficacious social on-target restriction is of the utmost importance, so that people can safely participate in medical check-up programs and come to hospitals when they have upper gastrointestinal symptoms. 
a The ratio of gastrectomy in 2020 to that in 2019 .

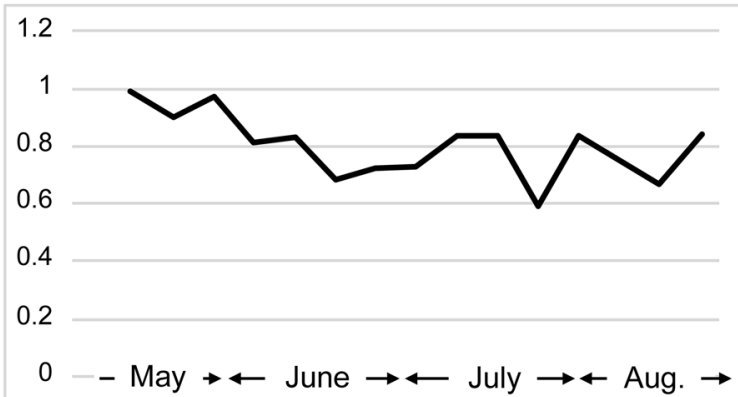

C The ratio of gastrectomy in 2020 to that in 2019 , stratified by the location of institution.

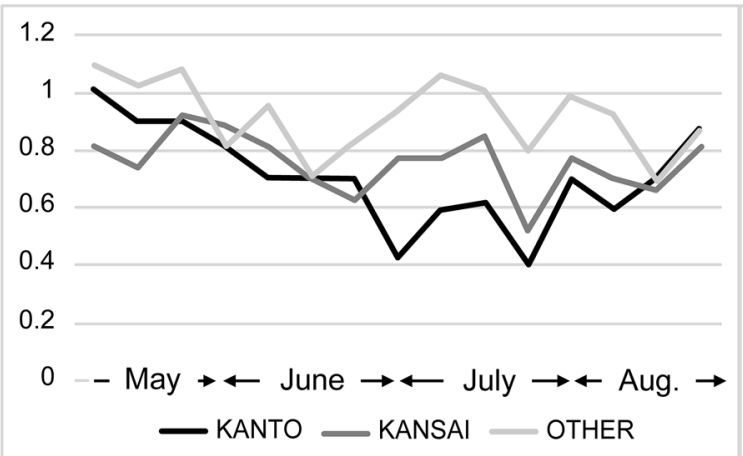

b The ratio of gastrectomy in 2020 to that in 2019 , stratified by the type of institution.

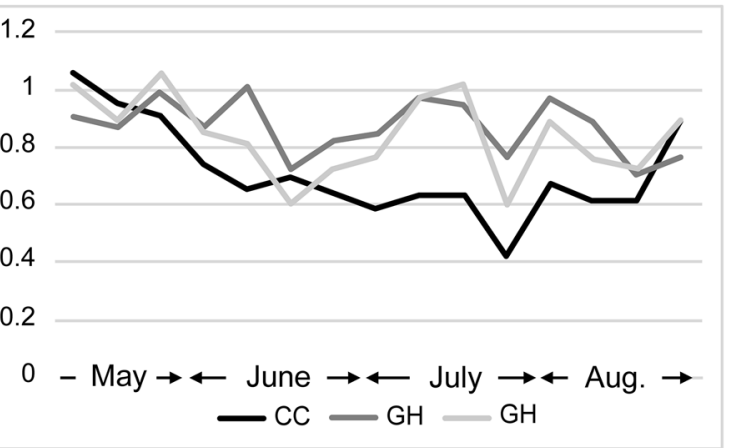

d The ratio of gastrectomy in 2020 to that in 2019 , in institutions located in Tokyo.

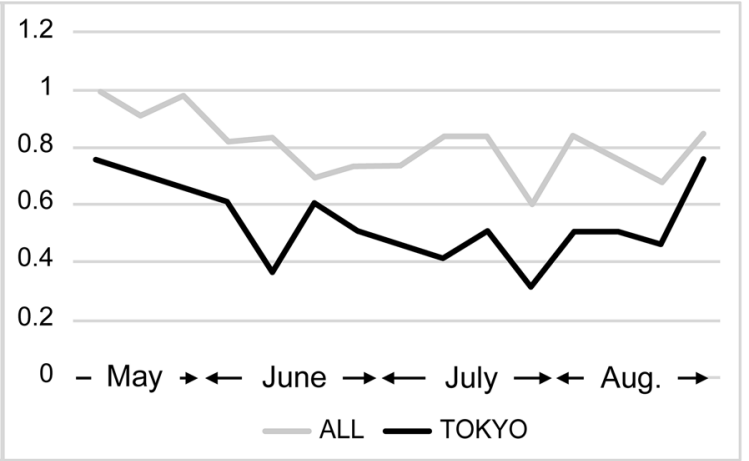

Fig. 5 The ratio of gastrectomy in 2020 to that in 2019 in each week (a). The effect of COVID-19 on the number of gastrectomies was stratified by the type of institution (b) and the location of institution (c). It was also calculated with hospitals in Tokyo (d)

Supplementary Information The online version contains supplementary material available at https://doi.org/10.1007/s00595-021-02329-y.

Funding This study was supported in part by the Japan Agency for Medical Research and Development (20ck0106591h0001).

\section{Declarations}

Conflict of interest The authors declare no conflicts of interest in association with the present study.

Ethical standards All procedures performed were in accordance with the ethical standards of the responsible committee on human experimentation (institutional and national) and with the Helsinki Declaration of 1964 and later versions.

\section{References}

1. Suh YS, Yang HK. Screening and early detection of gastric cancer: east versus West. Surg Clin North Am. 2015;95(5):1053-66.

2. Katai H, Ishikawa T, Akazawa K, Isobe Y, Miyashiro I, Oda I, et al. Five-year survival analysis of surgically resected gastric cancer cases in Japan: a retrospective analysis of more than 100,000 patients from the nationwide registry of the Japanese Gastric Cancer Association (2001-2007). Gastric Cancer. 2018;21(1):144-54.

3. Hamashima C, Systematic Review Group and Guideline Development Group for Gastric Cancer Screening Guidelines. Update version of the Japanese Guidelines for Gastric Cancer Screening. Jpn J Clin Oncol. 2018;48(7):673-83.

4. Huang C, Wang Y, Li X, Ren L, Zhao J, Hu Y, et al. Clinical features of patients infected with 2019 novel coronavirus in Wuhan. China Lancet. 2020;395(10223):497-506.

5. Nicola M, Alsafi Z, Sohrabi C, Kerwan A, Al-Jabir A, Iosifidis $\mathrm{C}$, et al. The socio-economic implications of the coronavirus pandemic (COVID-19): a review. Int J Surg. 2020;78:185-93.

6. Watanabe M. The COVID-19 pandemic in Japan. Surg Today. 2020;50(8):787-93.

7. Mori M, Ikeda N, Taketomi A, Asahi Y, Takesue Y, Orimo T, et al. COVID-19: clinical issues from the Japan Surgical Society. Surg Today. 2020;50(8):794-808.

8. Bresadola V, Biddau C, Puggioni A, Tel A, Robiony M, Hodgkinson J, Leo CA. General surgery and COVID-19: review of practical recommendations in the first pandemic phase. Surg Today. 2020;50(10):1159-67.

9. Shi YJ, Wang H. Progress in the diagnosis and treatment of COVID-19 and the role of surgeons in the front line of the pandemic. Surg Today. 2020;50(11):1544-8.

10. Zhou F, Yu T, Du R, Fan G, Liu Y, Liu Z, et al. Clinical course and risk factors for mortality of adult inpatients with 
COVID-19 in Wuhan, China: a retrospective cohort study. Lancet. 2020;395(10229):1054-62.

11. Irisawa A, Furuta T, Matsumoto T, Kawai T, Inaba T, Kanno A, et al. Gastrointestinal endoscopy in the era of the acute pandemic of coronavirus disease 2019: recommendations by Japan gastroenterological endoscopy society (Issued on April 9th, 2020). Dig Endosc. 2020;32(5):648-50.

12. Kuniya T. Evaluation of the effect of the state of emergency for the first wave of COVID-19 in Japan. Infect Dis Modell. 2020;5:580-7.

13. Tzeng CD, Teshome M, Katz MHG, Weinberg JS, Lai SY, Antonoff MB, et al. Cancer surgery scheduling during and after the COVID-19 first wave: the MD anderson cancer center experience. Ann Surg. 2020;272(2):e106-11.

14. Takahari D, Shinozaki E, Wakatsuki T, Ooki A, Ozaka M, Suzuki $\mathrm{T}$, et al. Managing a gastrointestinal oncology practice in Japan during the COVID-19 pandemic: single institutional experience in the Cancer Institute Hospital of Japanese foundation for cancer research. Int J Clin Oncol. 2020;26(2):335-44.

15. Wahed S, Chmelo J, Navidi M, Hayes N, Phillips AW, Immanuel A. Delivering esophago-gastric cancer care during the COVID19 pandemic in the United Kingdom: a surgical perspective. Dis Esophagus. 2020;33(9):doaa091.

Publisher's Note Springer Nature remains neutral with regard to jurisdictional claims in published maps and institutional affiliations. 